\title{
Structured Light System on Mars Rover Robotic Arm Instrument
}

\author{
Pedersen, David Arge Klevang; Liebe, Carl Christian; Jørgensen, John Leif
}

Published in:

IEEE Transactions on Aerospace and Electronic Systems

Link to article, DOI:

10.1109/TAES.2018.2874125

Publication date:

2019

Document Version

Peer reviewed version

Link back to DTU Orbit

Citation (APA):

Pedersen, D. A. K., Liebe, C. C., \& Jørgensen, J. L. (2019). Structured Light System on Mars Rover Robotic Arm Instrument. IEEE Transactions on Aerospace and Electronic Systems, 55(4), 1612 - 1623.

https://doi.org/10.1109/TAES.2018.2874125

\section{General rights}

Copyright and moral rights for the publications made accessible in the public portal are retained by the authors and/or other copyright owners and it is a condition of accessing publications that users recognise and abide by the legal requirements associated with these rights.

- Users may download and print one copy of any publication from the public portal for the purpose of private study or research.

- You may not further distribute the material or use it for any profit-making activity or commercial gain

- You may freely distribute the URL identifying the publication in the public portal

If you believe that this document breaches copyright please contact us providing details, and we will remove access to the work immediately and investigate your claim. 


\title{
Structured Light System on Mars Rover Robotic
}

\section{Arm Instrument}

\author{
David Arge Klevang Pedersen*, Carl Christian Liebe**, John Leif Jørgensen* \\ *The Technical University of Denmark, DTU Space, Elektrovej 327, 2800 Kgs. Lyngby, Denmark \\ **Jet Propulsion Laboratory, California Institute of Technology, 4800 Oak Grove Dr., Pasadena, CA 91109, \\ USA
}

\begin{abstract}
A structured light system is used to position a scientific instrument mounted on a Mars rover robotic arm relative to a Mars surface sample. Fifty laser spots are projected on the Mars surface sample. The identification of individual laser spots, the centroiding algorithm and the calibration are discussed. Results show that the system measures the distance to the Mars surface sample more accurate than $\mathbf{5 0}$ microns at a nominal operating distance of $\mathbf{3 0}$ mm.
\end{abstract}

Index Terms - CCD, laser, Mars 2020, Structured light, Triangulation $^{1}$

\section{INTRODUCTION}

The Planetary Instrument for X-ray Lithochemistry (PIXL) is a Micro-focus X-Ray Fluorescence (Micro-XRF) instrument for measuring fine scale chemical variations in rocks and soils on the Martian surface. It has been selected for the Mars 2020 rover science payload [1]. PIXL can measure elemental chemistry of tiny features observed in rocks, such as individual sand grains, veinlets, cements, concretions and crystals $[2,3]$.

The instrument is mounted on the rover robotic arm and must be located $30 \mathrm{~mm}+/-0.5 \mathrm{~mm}$ from a surface sample to be examined, for the 100 micron diameter X-ray spot to be focused. The sample would typically be abraded prior to investigation by PIXL. The abraded area would be relatively flat with an unknown topography of the surrounding area. The existing Mars 2020 rover robotic arm design does not have sensor instrumentation to position the PIXL instrument accurately relative to the Mars surface sample. Therefore, the structured light subsystem has been added to the PIXL instrument. The objective of the structured light system is twofold, 1) Perform a distance measurement of the instrument relative to the Mars surface sample for the X-ray measurement and 2) Perform hazard detection while the robotic arm is approaching and positioning the instrument relative to the Mars surface sample. An artist's conception

\footnotetext{
${ }^{1}$ (c) 2018. All rights reserved.
}

of the Mars Rover performing measurements with a turret mounted instrument guided by the robotic arm is shown in Figure 1.

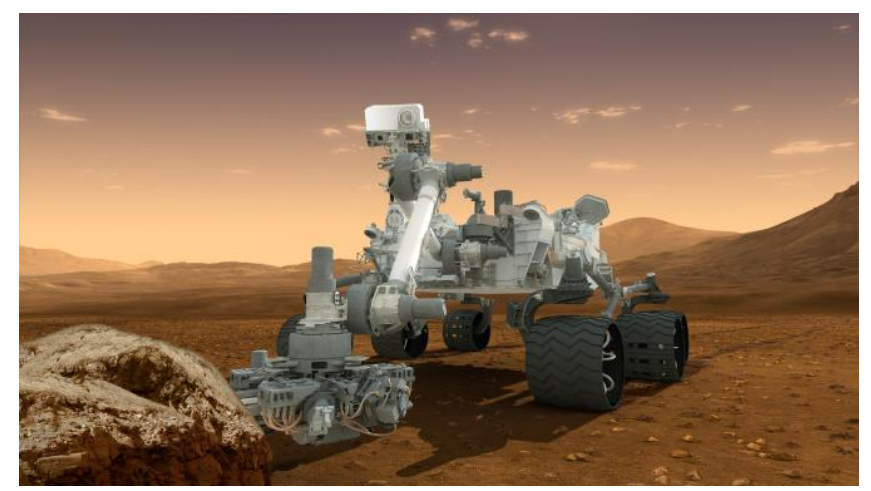

Figure 1: Artist conception of the rover taking a measurement with a turret mounted instrument on the Mars Rover Robotic Arm. (Credit NASA/JPL)

Sensors used for distance measurements have a wide range of applications where the technology of use depends on the required accuracy and range of operation. It is possible to measure distance passively (assuming an illuminated target) by methods based on stereo vision [4]; depth from focus [5]; or coded aperture masks [6]. By active means, distance can be measured by time of flight of a pressure pulse in air [7] or water [8]; microwave radars [9]; interfering laser beams [10], phase modulating lasers [11]; amplitude modulating lasers [12]; laser pulse time of flight [12]; and triangulation [13] can be used for distance measurement. In this specific application, the system is required to operate at night, and therefore, an active sensing technology was chosen. Furthermore, it is required that the system is able to measure distance to some small points to sub $\mathrm{mm}$ accuracy. The technology that is best suited for this is utilizing a laser (as opposed to sonar or micro wave).

Technologies for measuring distance utilizing laser beams are evolving fast. Traditionally, distance measuring sensors has been measuring the distance to a single point. However, 
technologies are evolving into making 3D images with distance measurements to many points in the field of view. Examples are the Microsoft Kinect sensor [14], time of flight cameras [15] [16], and structured light systems [17] [18]. In this specific application, a measurement was required to multiple points. Therefore, a traditional point to point laser distance measurement technology was not feasible. At the same time, the instrument already included a camera for the scientific interpretation of the X-ray measurements. Implementing a distance measuring system utilizing structured light with multiple laser beams, was therefore the preferred choice, adding only small extra mass and complexity to the existing instrument.

Distance measurement is an active area of research and development in the aerospace industry. Distance measurement sensors are being used for rendezvous and docking applications [19] and for safe landing applications [20]. Distance estimation instrumentation is also used for Mars rover navigation [4] [21]. A structured light system has also been used for rover navigation on NASA's Mars Pathfinder rover [22]. This is the only structured light system on a planetary rover to the knowledge of the authors. However, this is the first time that an optical distance sensor has been used to position a scientific instrument to submillimeter accuracy for a robotic non-terrestrial application. Past Mars missions have also placed scientific instruments in the proximity of targets on the surface. This was accomplished utilizing stereo vision based on forward looking cameras mounted on the rover [23]. However, this method is only accurate to $\sim 1 \mathrm{~cm}$ and not accurate enough for the PIXL instrument.

The contribution of this paper is to describe a prototype structured light sensor that must operate on the surface of Mars. It must operate more than $80^{\circ} \mathrm{C}$ colder than it was calibrated at. Also, it must operate in direct sunshine with a deposited layer of dust. Simultaneously, there are severe limitations on mass and power and the system must enable scientific image recordings of the PIXL instrument.

A diagram of the structural light system is shown in Figure 2. The illumination consists of 2 high powered $(200 \mathrm{~mW})$ NIR diode lasers. Each laser is split up in 15 or 35 laser beams in a diffraction grading. This way it is possible to measure the distance to 50 points on the Mars surface sample. A modified star tracker, the microASC [24], with abundance of heritage from missions like Juno, GFO, MMS and Swarm, is used to detect the laser spots. The microASC consists of a Digital Processing Unit (DPU) and a Camera Head Unit (CHU) with a monochrome CCD chip. For this application the standard lens of the CHU is replaced by a miniature lens with a small aperture to ensure focus over a large operational range. Also a spectral filter is added to the optical chain. The filter primarily transmits light at the laser wavelength, but it also transmits light at other wavelengths so it is possible to acquire an image of the Mars surface sample as context for interpreting the scientific X-ray measurements. A bank of LEDs is also used to illuminate the Mars sample for night time operation. However, the LEDs are not used for the structural light application. The required accuracy for the structured light system is given in Table 1, with a nominal operational standoff distance of 30 $\mathrm{mm}$, referenced to the tip of the X-ray optics.

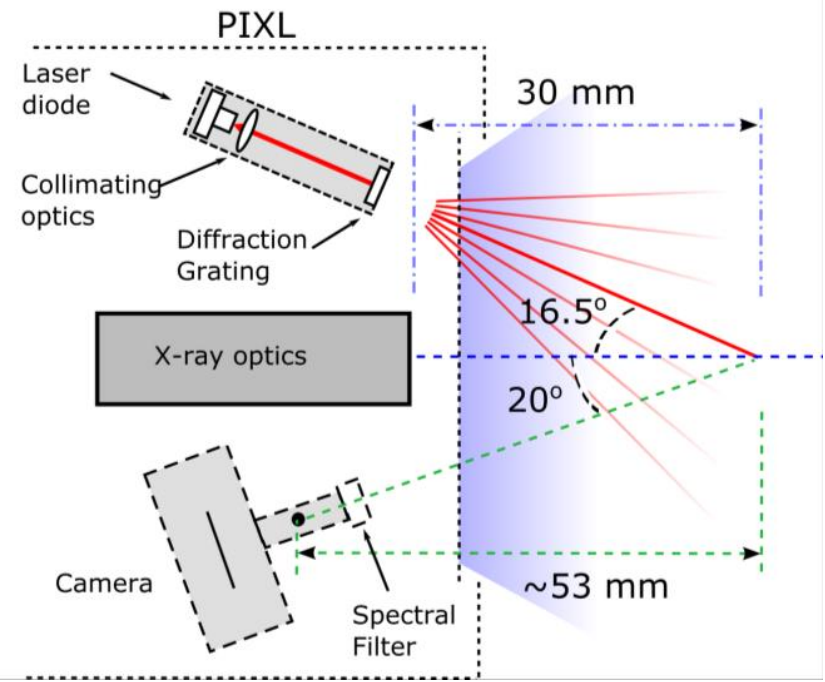

Figure 2: Diagram of the structured light system together with the $\mathrm{X}$ ray optics of the PIXL instrument. The system consists of two collimated laser sources split into 15 and 35 beams, a CCD based camera with a spectral pass band filter and a bank of 40 LEDs. For simplicity only one laser source is illustrated.

Table 1: Accuracy requirements for the structured light system, specified within a standoff distance interval.

\begin{tabular}{ll}
\hline \hline Standoff distance & $3 \sigma<2.0 \mathrm{~mm} @ 10-20 \mathrm{~mm}$ \\
$3 \sigma$ & $<0.7 \mathrm{~mm} @ 20-40 \mathrm{~mm}$ \\
& $3 \sigma<2.0 \mathrm{~mm} @ 40-100 \mathrm{~mm}$ \\
\hline Lateral position & $3 \sigma<1.0 \mathrm{~mm} @ 20-25 \mathrm{~mm}$ \\
& $3 \sigma<0.5 \mathrm{~mm} @ 25-35 \mathrm{~mm}$ \\
& $3 \sigma<0.5 \mathrm{~mm} @ 35-40 \mathrm{~mm}$ \\
\hline \hline
\end{tabular}

A conceptual error budget for a flight implementation is shown in Table 2. The error budget accounts for thermal effects, laser wavelength shifts due to temperature, effects due to gravity release, calibration residuals, and centroiding errors etc. The error budget shown in Table 2 is a summary of a simple model of the structural light system with 19 individual error terms. A full description of all error terms in the error budget is outside the scope of this paper. However, a single term in the error budget is discussed in which shows the methodology for generating the error budget. Figure 3 shows a simple model of the structure light system with the camera and laser connected $t$ a mechanical structure that is equivalent to $40 \mathrm{~mm}$ of aluminum structure. The system is calibrated at $20 \mathrm{C}$, but when operating at the surface of Mars the temperature is $-60 \mathrm{C}$. Due to the thermal expansion coefficient of aluminum, the structure dimension between the laser and the camera will shrink to $39.91 \mathrm{~mm}$ at the surface of Mars. This will move the orientation of the laser dot 0.08 degrees.. Since the structure light system calibration assumes the camera and laser is still separated by 
$40 \mathrm{~mm}$, it calculates the distance to the target to $53.23 \mathrm{~mm}$ (in reality the software will utilize calibrations from different temperatures.). An argument has also been generated for the remaining 18 error contributions, where an assumed distortion is converted into a distance error and added up (independent error contributions are RSS but some thermal contributions are added or subtracted).

Table 2: Error budget for flight implementation of structured light system.

\section{Error due to thermal motion}

$0.4 \mathrm{~mm}$

Translations in the camera, translations in the structured light illuminator, translations in the structure holding the structured light illuminator and the camera, change in structured light illuminator orientation, change in camera orientation, change in orientation between camera and structured light illuminator, change in camera field of view.

\section{Calibration Accuracy}

Centroiding accuracy during calibration, accuracy of distance calibration (laser tracker accuracy), stability of laser tracker targets, accuracy of establishing coordinate system during alignment and when installing the structured light system on Mars rover.

\section{Centroiding Accuracy}

Centroiding noise, centroiding systematic errors, centroid error due to target being at an angle, centroid error due to changes in albedo of target, centroiding error due to laser speckles.

\section{Wavelength change in laser}

Laser wavelength changes $0.3 \mathrm{~nm} / \mathrm{C}$. The wavelength change will affect angular divergence in the diffractive grating. Assumes that the temperature of the laser is known to 10C.

$\begin{array}{ll}\text { Gravity Release } & 0.1 \mathrm{~mm} \\ \text { Change in mechanical configuration when gravity is only } 0.6 \mathrm{G} & \end{array}$
and coming from different orientation.

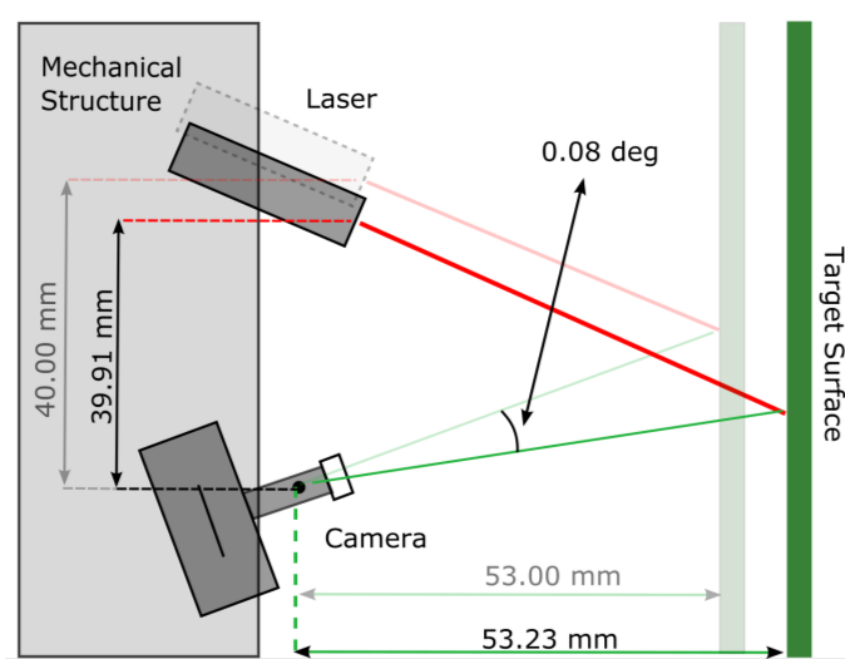

Figure 3: Illustration of how the shrinking of the mechanical structure affects the distance measurement. The baseline between the camera and the laser is displaced from $40 \mathrm{~mm}$ to $39.91 \mathrm{~mm}$, resulting in an angular shift of the laser dot, as seen from the camera. With the calibration conducted at room temperature, the perceived distance at $-60 \mathrm{C}$ will be $53.23 \mathrm{~mm}$.

To verify the combined system performance of the camera and structured light, a demonstration system has been constructed. Experiments are conducted in order to verify the centroiding and system calibration, in particular, considering worst case behavior and performance on representative rock samples. An overview of the paper is as follows:

Section II of this paper discusses the radiometry of the structured light system. Section III discusses the algorithm for identifying the individual laser beam corresponding to the 50 laser spots in the image. Section IV will discuss the centroiding algorithm used in the structured light system. In Section V, the algorithm for converting the centroid measurements into distances is discussed. Section VI will discuss the calibration of the structured light system. Finally, Section VII will discuss test results from field testing with the demonstration system.

\section{SAMPLE ILLUMINATION RADIOMETRY}

The instrument is designed for operation under all illumination conditions, i.e. night time operation and fully sun illuminated conditions. For night time operations, a bank of LEDs is used to illuminate the Mars surface sample to generate context images for the scientific interpretation of the X-ray measurements.

The total radiant exitance of a black body is described by Stefan-Boltzmann Law [25]:

$$
M=\varepsilon \sigma T^{4},
$$

where $T$ is the temperature of the black body, $\varepsilon$ is the emissivity (unity for black bodies) and $\sigma$ the constant of proportionality, given as:

$$
\sigma=\frac{2 \pi^{5} k^{4}}{15 c^{2} h^{3}}
$$

where $c$ is the speed of light in vacuum, $k$ is the Boltzmann's constant and $h$ is Planck's constant. The total solar irradiance at Mars is thus given by:

$$
E_{\text {sun }}=M \frac{R_{\text {sun }}^{2}}{D_{\text {mars }}^{2}}
$$

where $R_{\text {sun }}$ is the radius of the Sun and $D_{\text {mars }}$ is the distance from the Sun to Mars. With the relatively high elliptical orbit of Mars the distance to the Sun varies considerably. The maximum irradiance at perihelion is $717 \mathrm{~W} / \mathrm{m}^{2}$ and minimum at aphelion $493 \mathrm{~W} / \mathrm{m}^{2}$. Averaging the distance over the mean anomaly gives an irradiance of $583 \mathrm{~W} / \mathrm{m}^{2}$.

The Martian atmosphere is approximated to be transparent to radiation in the visual spectrum [26].

The laser beam has an elliptical Gaussian profile of $0.5 \mathrm{~mm}$ $(3 \sigma)$. The laser is derated to $50 \mathrm{~mW}$ and assuming a 
diffractive grating transmission of 0.3 , optics transmission of 0.5 and splitting the two laser beams into 35 and 15 collimated beams, results in an irradiance on the target surface of $E_{\text {laser }}=546 \mathrm{~W} / \mathrm{m}^{2}$ and $E_{\text {laser }}=1273 \mathrm{~W} / \mathrm{m}^{2}$. The intensity of the split laser beams varies up to $30 \%$ relative to the brightest beam. For this analysis it is assumed that the laser spots have equal brightness. The spectral width of the laser is $3 \mathrm{~nm}$.

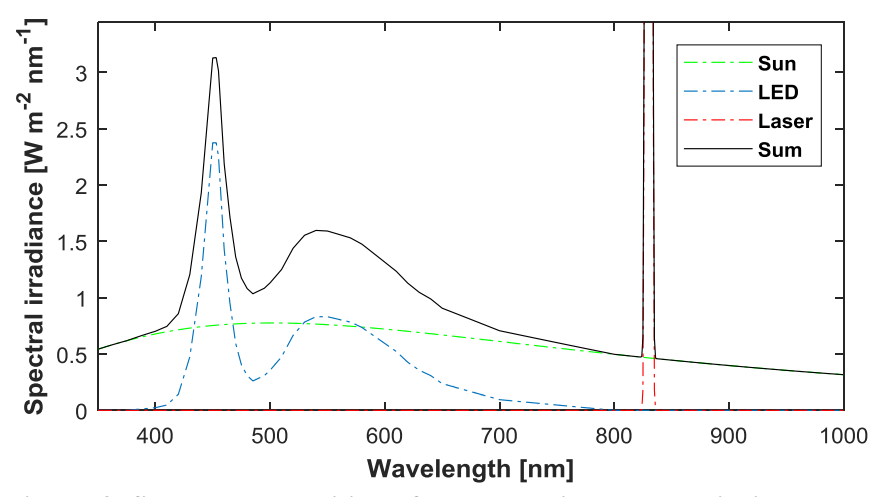

Figure 4: Spectral composition of the three light sources incident on the Martian target surface. The peak of the light from the laser source is not visible as it is several orders of magnitude larger than the other light sources.

A demonstration LED bank consists of 40 white LEDs each with $1.8 \mathrm{~mW} / \mathrm{sr}$ radiant intensity. At a distance of $20 \mathrm{~mm}$ to the target surface and a view angle of $120^{\circ}$ the irradiance from a single LED is $4.4 \mathrm{~W} / \mathrm{m}^{2}$. With 40 LEDs the total irradiance from the LED bank approximates to $E_{L E D}=$ $211 \mathrm{~W} / \mathrm{m}^{2}$.

At the nominal standoff distance $(53 \mathrm{~mm}$ from the camera pinhole) the total spectral irradiance upon the Martian target surface is shown in Figure 4.

The global optical properties of the Martian surface have been studied [27] [28]. However, on a microscopic scale, the structured light system will encounter many different types of Mars rocks. The surfaces may be clean, weathered, covered in dust or abraded. A series of basaltic rocks, mudstones, sandstones, conglomerates and stromatolites were examined, that are representative for what the rover will encounter on Mars [2] [29] [30] [31] [32]. To cover a variety of rock types a Lambertian surface is assumed. The radiant intensity of the radiation reflected off such a surface is given by [33]

$$
I(\lambda)=\frac{E(\lambda) \alpha(\lambda) A_{s}}{\pi}
$$

where $A_{s}$ is the surface area covered by the sensors Field of View, $\alpha(\lambda)$ is the albedo of the surface. In this analysis an average albedo of 0.25 [34] is adopted as the spectral albedo will vary depending on the actual surface material. Given the solid angle $\Omega$ covered by the lens aperture towards the surface, the total power reaching the aperture is

$$
P_{a}(\lambda)=\frac{E(\lambda) \alpha A_{s} \Omega}{\pi} .
$$

With the solid angle given by the aperture area and the distance to the surface $\Omega=A_{a} / D_{s}^{2}$, and the surface area $A_{s}$ given by the CCD pixel size, focal length and distance to the surface $A_{s}=\frac{p i x^{2}}{f^{2}} D_{s}^{2}$, Equation (5) reduces to

$$
P_{a}(\lambda)=\frac{E(\lambda) \alpha(\lambda) p i x^{2} A_{a}}{\pi f^{2}}
$$

A narrow pass band filter designed to transmit in a $\pm 10 \mathrm{~nm}$ pass band around $832 \mathrm{~nm}$ and attenuate other frequencies to $10 \%$ of the original signal is mounted in front of the camera optics. This increases the signal to background ratio of the laser signal. The number of photoelectrons detected by the sensor is given by

$$
N_{e^{-}}=\frac{Q E(\lambda) \cdot B_{B P}(\lambda) \cdot B_{L}(\lambda) \cdot P_{a}(\lambda)}{E_{\text {photon }}(\lambda)}
$$

where $Q E$ is the quantum efficiency of the $\mathrm{CCD}, B_{B P}$ and $B_{L}$ are the transmission of the band-pass filter and camera lens optics and $E_{\text {photon }}$ is the photon energy, given by

$$
E_{\text {photon }}=\frac{h \cdot c}{\lambda}
$$
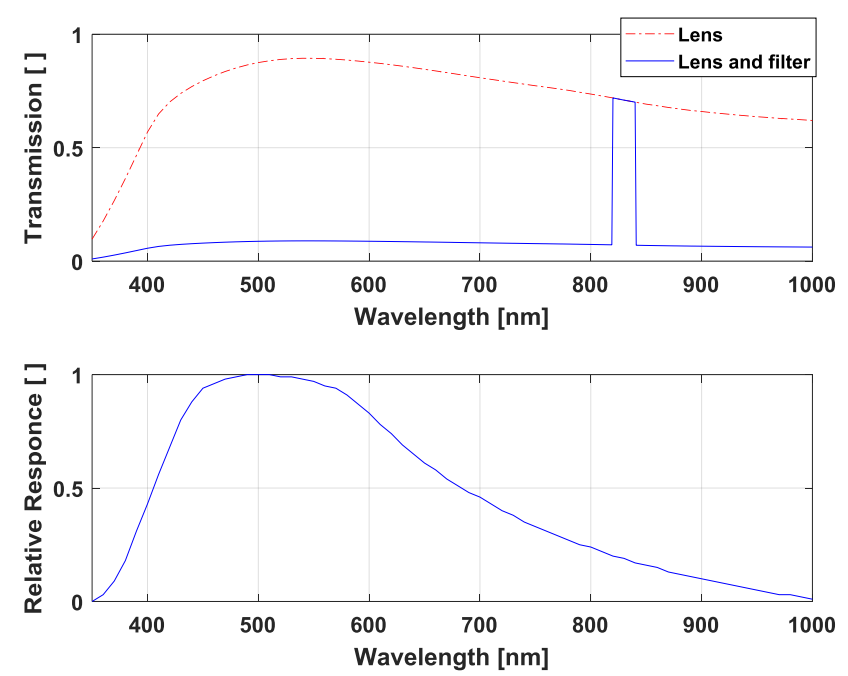

Figure 5: Top: Transmission of the lens optics together with the band pass filter. Bottom: Relative spectral sensitivity of the CCD.

Figure 5 shows the optical transmission of the lens and the filter together with the spectral sensitivity characteristics of the CCD which has a QE of 65\% at the peak around 500 $\mathrm{nm}$. Integrating over the wavelength, $\lambda$, gives the total number of photoelectrons. With the optical transmission of the lens + filter, QE of the CCD and camera parameters given in Table 3, the total number of photoelectrons for each of the three light sources is calculated and listed in Table 4. 
Table 3: Parameters for the camera system. It is observed that the f/\# of the lens is 16. The reason for this is that the system must acquire focused images over a wide range of focal distances.

\begin{tabular}{ll}
\hline \hline Focal length & $12 \mathrm{~mm}$ \\
F-number & 16 \\
Pixel size & $8.6 \times 8.3 \mu \mathrm{m}$ \\
CCD resolution & $752 \times 580$ pixels
\end{tabular}

Table 4: Total number of photoelectrons detected by a single pixel of the CCD at the nominal distance of $53 \mathrm{~mm}$.

\begin{tabular}{ll}
\hline \hline Sun & $6.17 e 5 \mathrm{e}^{-} / \mathrm{s}$ \\
Laser & $8.09 e 6 \mathrm{e}^{-/ \mathrm{s}}$ \\
LED bank & $3.90 e 5 \mathrm{e}^{-/ \mathrm{s}}$
\end{tabular}

Noise contributions for the measured image intensities are: dark current, read noise, quantization error and shot noise. The dominant error depends on the lighting conditions. The described system will have a full well capacity on the order of $10^{5} \mathrm{e}^{-}$, dark current of $2 \mathrm{e}^{-} / \mathrm{s}$ at $10^{\circ} \mathrm{C}$, read noise of $19 e^{-}$and an 8 bit $\mathrm{AD}$ converter, resulting in a quantization error of $\frac{10^{5}}{2^{8} \sqrt{12}}=113 \mathrm{e}^{-}$[35]. The shot noise will a reach a maximum of $\sqrt{10^{5}}=316 \mathrm{e}^{-}$which is the limiting factor of the camera system achieving a combined $\mathrm{S} / \mathrm{N}$ of $\frac{10^{5}}{\sqrt{19^{2}+113^{2}+316^{2}+2^{2}}}=297$.

In conclusion, it is possible to achieve sufficient laser illumination with the described system with $\frac{10^{5} e^{-}}{6.17 e 5 e^{-} / \mathrm{s}+8.09 e 5 e^{-} / \mathrm{s}}=11.5 \mathrm{~ms}$ exposure time at a nominal distance under sunlit conditions. An example of an abraded Saddleback Basalt target illuminated by the Sun and single laser spot is shown in Figure 6.

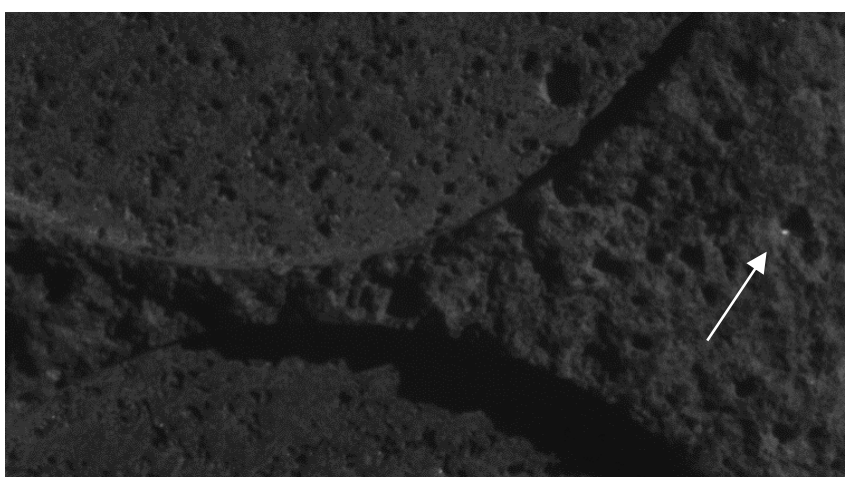

Figure 6: Image of abraded Saddleback Basalt surface illuminated by the Sun (late afternoon), corresponding to the expected power on the Martian surface, and a single laser spot. The image is acquired with an exposure time corresponding to $40 \mathrm{~ms}$ at a standoff distance of 107 mm.

\section{LASER SPOT IDENTIFICATION ALGORITHM}

One problem, that all multiple beam structured light systems faces, is the following: In an image of multiple laser spots being projected onto a surface of unknown topography, which laser spot is which? This Section will describe the algorithm used to establish the identity of the laser dots.
The structured light system consists of 2 individual illuminators. They are not turned on at the same time. The primary reason for this is to simplify the laser spot identification. The 2 structured light illuminators represents 1) a dense grid to establish distance to the Mars sample, close to where the X-ray beam is intercepting and 2) a sparse laser grid, used for hazard detection during approach and positing of the PIXL instrument by the robotic arm. A picture of the two spot patterns at nominal distance is shown in Figure 7 and Figure 8.

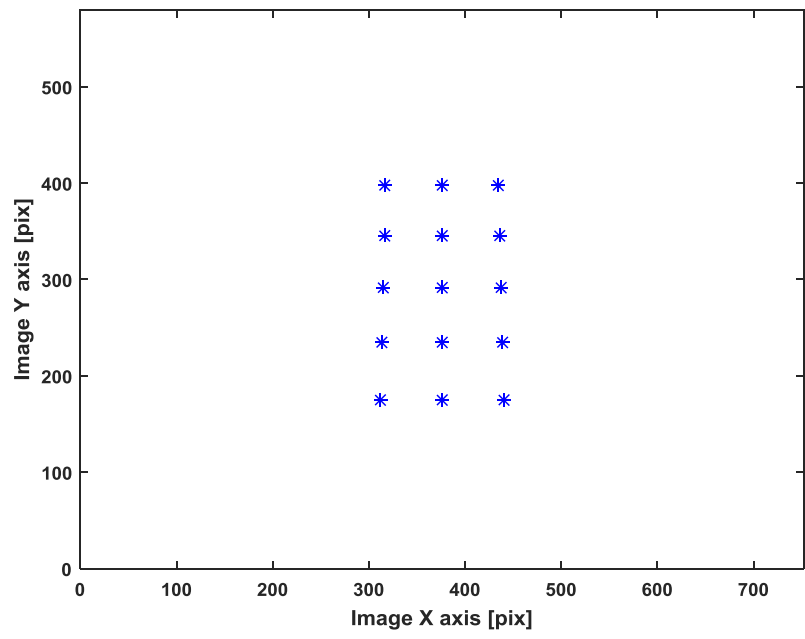

Figure 7: The dense laser spots at nominal $(30 \mathrm{~mm})$ distance.

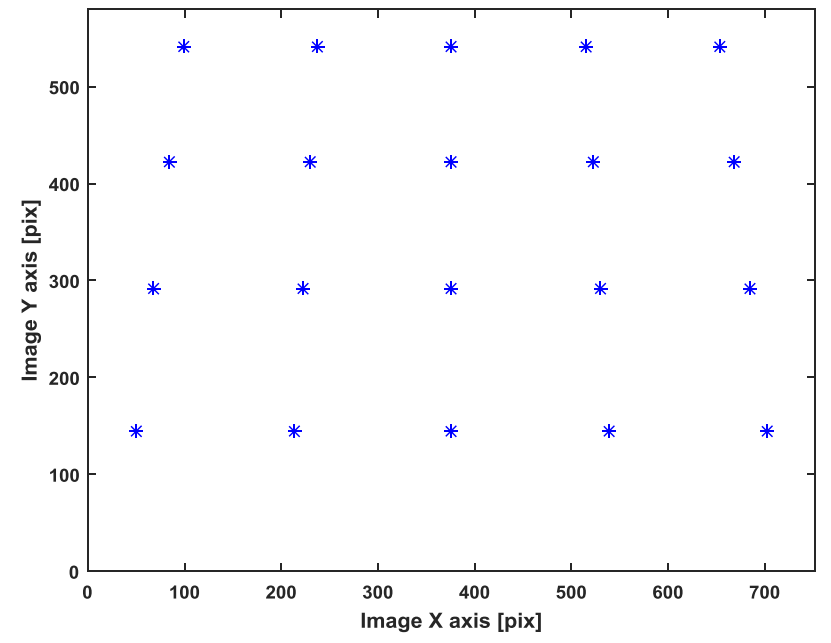

Figure 8: The sparse laser spots at nominal $(30 \mathrm{~mm})$ distance.

When the distance to the surface is changed, the position of the laser dots are also changed due to the baseline difference between the laser and the camera. As an example, when the Mars sample is imaged continuously between the distances from 20 to $40 \mathrm{~mm}$, the motions of the different laser spots are as shown in Figure 9. This is the range of motion where this dense structured light system has to operate. The coordinates formed from the spots lie on straight lines, analogous to epipolar lines in stereo vision [36]. In Figure 10 is shown the laser spot positions of the sparse array when the distances change from $20 \mathrm{~mm}$ to $100 \mathrm{~mm}$.

During calibration, 50 different images are generated (one of each laser spot). The individual images represent all possible 
positions in the image that this specific laser spot can be located at. Because there are errors and uncertainties associated with a measurement, the image for each laser spot is dilated 2 pixels. This accounts for the errors. As an example, the image for laser spot 6 (lower right corner in Figure 10) from the dense array is shown in Figure 11.

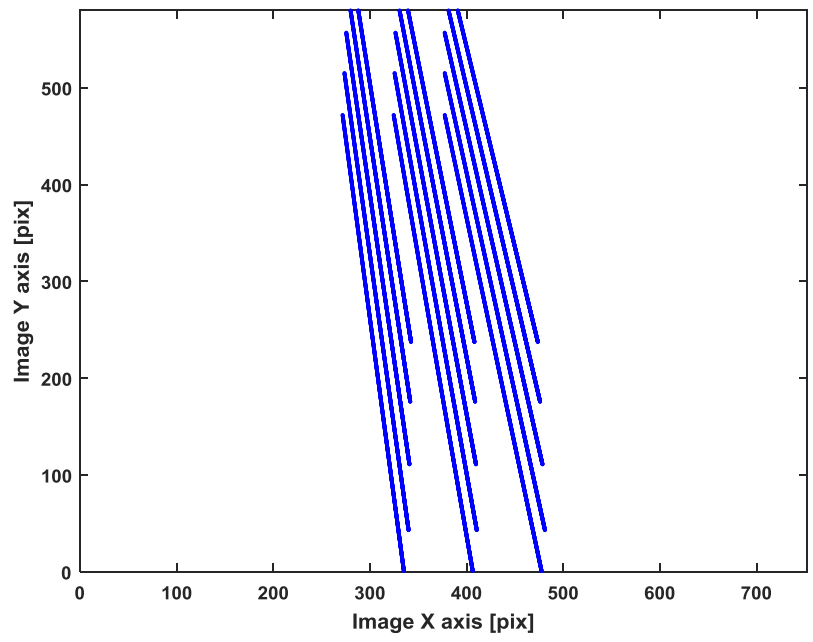

Figure 9: The trajectory of the 15 laser spots from the dense array, seen from the camera, as the distance is changed from 20 to $40 \mathrm{~mm}$.

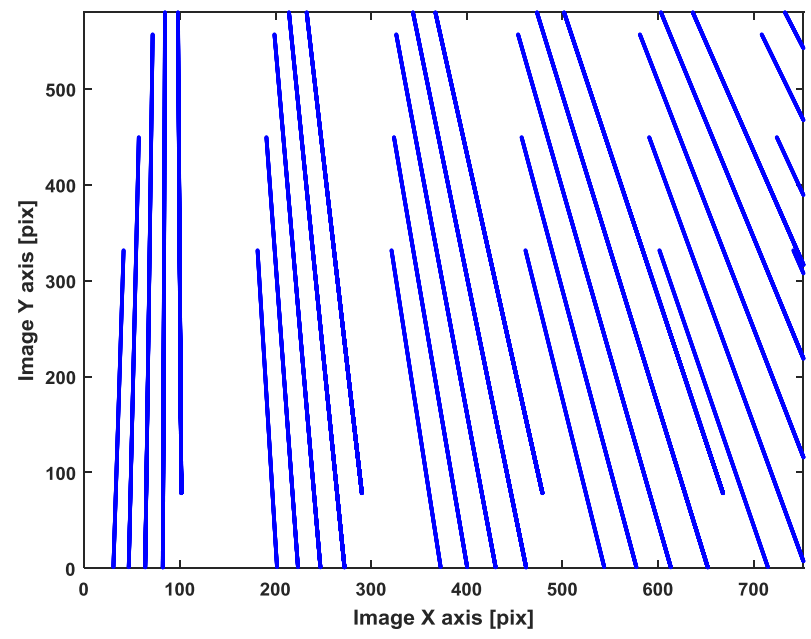

Figure 10: The trajectory of the 35 laser spots from the sparse array, seen from the camera, as the distance is changed from $20 \mathrm{~mm}$ to 100 mm.

When the 50 individual laser maps have been generated, the structured light system is ready to do spot identification. An example of an image that the structured light system could encounter is shown in Figure 12.

The algorithm operates by comparing the individual spots to the 50 individual spot maps. The spot is identified as the image where it has been registered at this specific position. This is illustrated in Figure 13. It is advantageous to design the system so there is no overlap between the individual spots (the described structured light system does not have overlap). In case there is an overlap, it will sometimes not be possible to uniquely identify the laser spot.

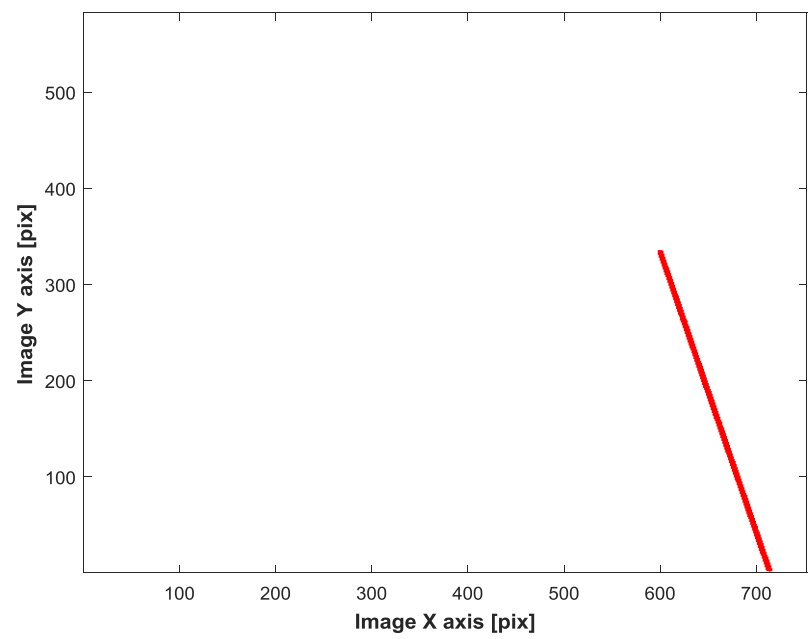

Figure 11: The location map where laser spot 6 can be located.

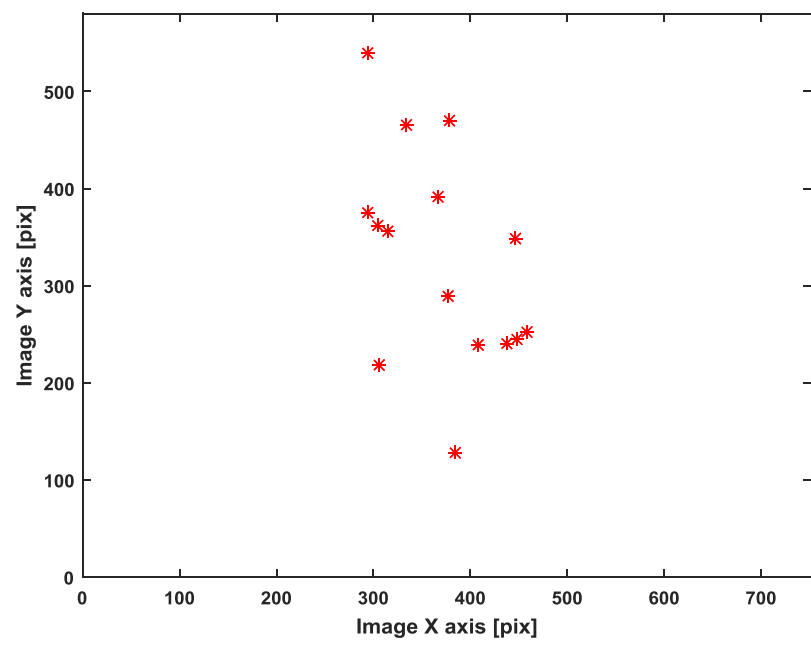

Figure 12: An example of an image that the structured light system could encounter. The system must be able to identify which dot belongs to which laser dot.

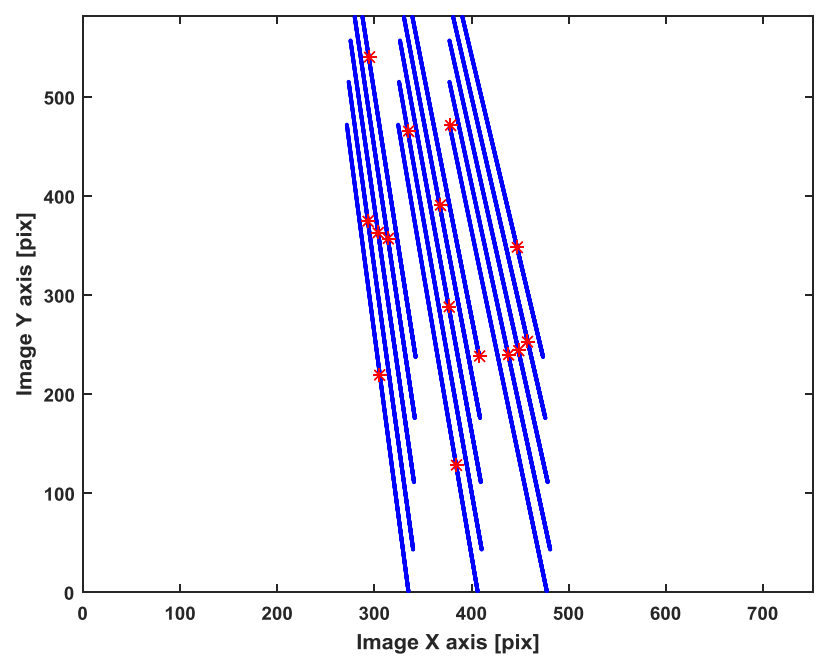

Figure 13: Image with the 15 individual laser maps (for the dense system) overlaid with the picture of the laser dots. It is observed it is easy to identify the individual spots.

\section{LASER SPOT CENTROIDING}

The output of the laser source is a collimated elliptic Gaussian profile. One method of centroiding would be to correlate or fit to an elliptical two dimensional Gaussian 
profile. However, due to the varying albedo and shape of the Martian surface the intensity profile will be distorted and deviate substantially from the original profile. Therefore an intensity weighted centroid is used to estimate the center of the laser spot. The moment is given by

$$
M_{p q}=\sum_{x} \sum_{y} x^{p} y^{q} W(x, y),
$$

where $p$ and $q$ define the moment order and $W$ the weighting. The weighting can be unity (binary images) or as in this case correspond to the intensity of a grayscale image. The centroid is given by the zeroth and first moments [37]

$$
\left[x_{c}, y_{c}\right]=\left[\frac{M_{10}}{M_{00}}, \frac{M_{01}}{M_{00}}\right] \text {. }
$$

Two images are captured to detect the centroid; one with the laser on and one with the laser off. Subtracting the two images leaves only the information from the laser.

To assess the accuracy of the centroid algorithm a couple of tests were conducted. The initial test, measuring a plane surface with a uniform albedo, is to quantify the centroid accuracy at nominal standoff distance with no surface properties affecting the result. The resulting centroid accuracy is 0.2901 pixels $(1 \sigma)$ with an elliptic laser spot of $10 x 15$ pixels. The exposure time is set so the laser spot is not overexposed imitating an autonomous exposure controlloop optimizing for the brightest of the visible spots, leaving remaining spots slightly underexposed.

A driving error term is the varying albedo: when the laser spot illuminates the boundary of two regions with high contrast, reducing the elliptical Gaussian profile to a semielliptical profile. Figure 14 shows the results when targeting a plane surface with a sharp transition between black and white regions, representing the worst case scenario. The two top plots show the $x$ and $y$ coordinate of the centroid as a function of the lateral translation of the target relative to the instrument cluster. A bias, just short of 4 pixels is introduced as the laser spot travels over the black/white boundary. The bias is present in both coordinates as the black/white boundary is not aligned to the CCD chip of the sensor. The bias of 4 pixels is close to the expected result when considering the elliptical profile of $10 \times 15$ pixels is reduced to a semielliptical profile. The bottom plot shows the relative distance estimate as a function of the translation of the target, in which the transition manifests itself as a $0.72 \mathrm{~mm}$ distance chance. It should be noted that this is a worst case scenario. For natural occurring targets, a maximum error of $0.4 \mathrm{~mm}$ has been assumed in the error budgets (distance estimation and calibrate the system is addressed later in this paper).
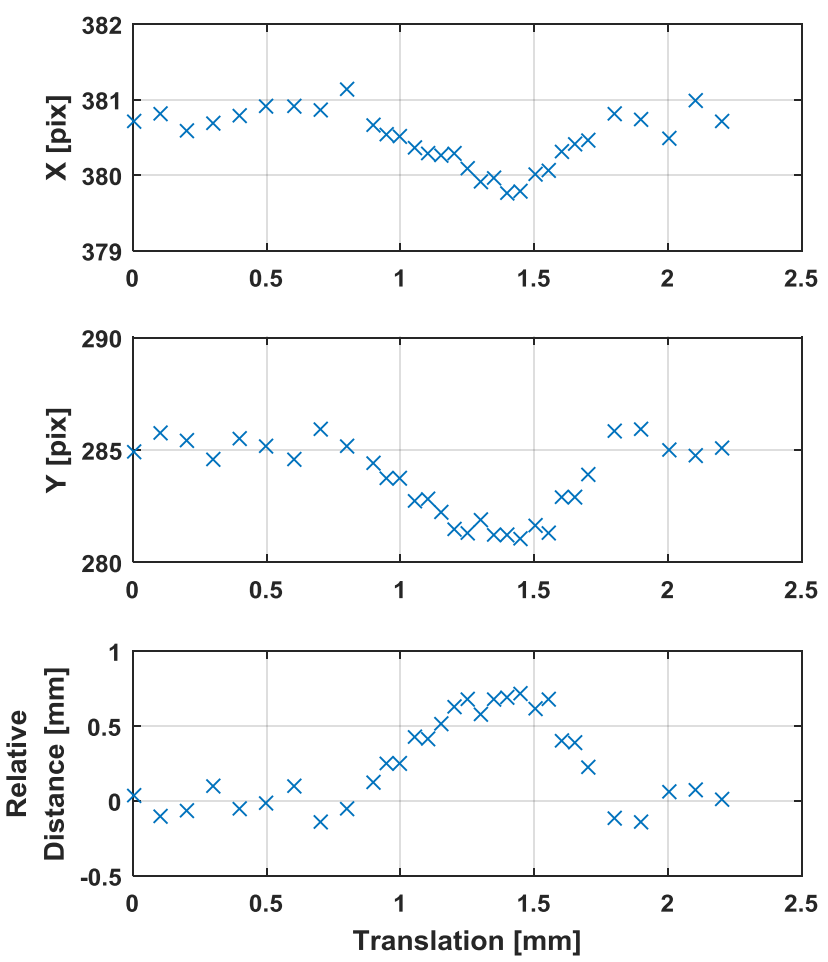

Figure 14: Test results using a target with a sharp transition of black and white background. The two top plots show the $x$ and $y$ centroid coordinates of a single laser spot as a function of the lateral translation of the target. The bottom plot shows the resulting relative distance as a function of the lateral translation of the target as the black/white boundary of the target is translated across the laser spot.
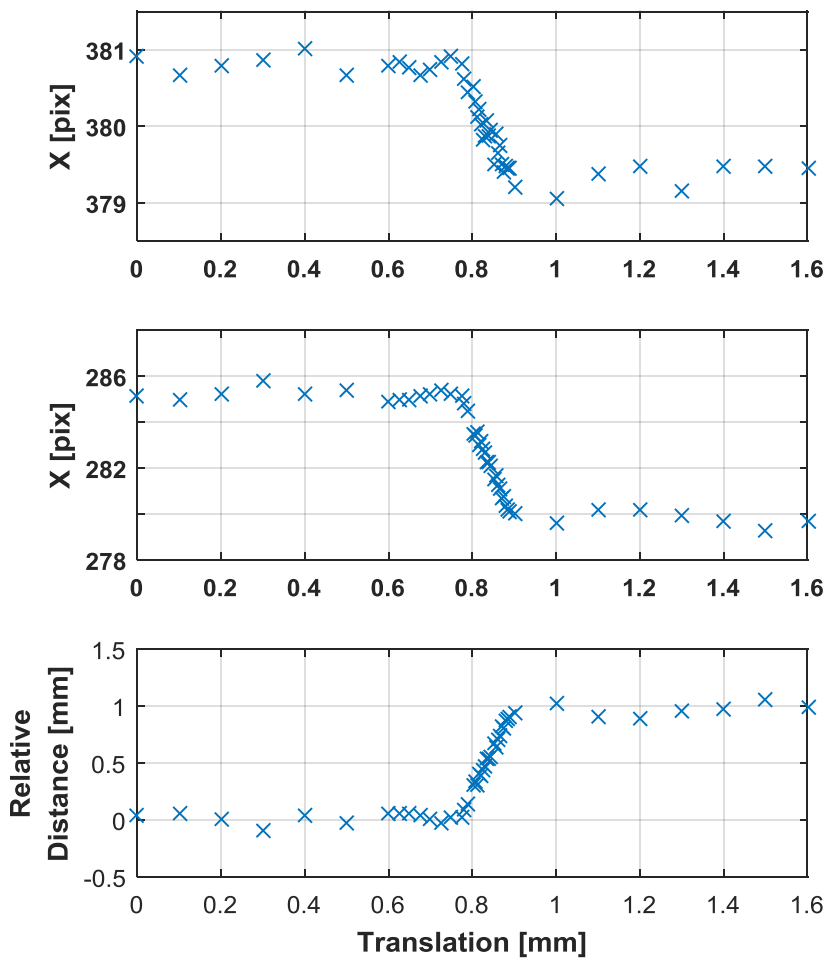

Figure 15: Test results using target with a delta height increment of $0.930 \mathrm{~mm}$. The two top plots show the $x$ and $y$ centroid coordinates of a single laser spot as a function of the targets lateral translation. The bottom plot shows the centroids lateral displacement as the boundary of the height increment is translated across the laser spot.

The local topography of the surface will also introduce a bias to the centroid measure, as the elliptical profile of the laser spot is distorted. To assess the resulting bias a simple situation is considered: Assuming a flat surface tilted 45 
degrees from the perpendicular alignment, the expected bias is in the order of 0.05 pixels at nominal standoff distance, due to the perspective and radiometric distortion (according to a computer simulation). This is not a significant error term and is thus neglected.

To understand how the centroid algorithm responds to sharp transitions of local topography, a test was conducted when measuring a surface with uniform albedo, and a step height increment of $0.930 \mathrm{~mm}$. The resulting $x$ and $y$ coordinate of the centroid, as well as the relative distance as a function of the lateral translation of the target is shown in Error! Reference source not found.. It is observed, the centroid has a linear transition between the two surfaces, resulting in a linear shift of the distance estimate close to the expected $0.930 \mathrm{~mm}$.

\section{DISTANCE MEASUREMENT}

The centroid of each laser spot makes it possible to calculate the distance. The individual laser measurements can be combined to estimate the plane of the abraded area or used separately for topography information or simply utilizing the single spot closest to the X-ray beam. A sketch of the structured light system is shown in Figure 16. The camera system is located at the equivalent pinhole of the camera. In a noiseless system the lasers trajectory and the centroid line of sight will intersect where the laser beam illuminates the targeted surface. Now, with noise inherent in the system, these will not intersect, thus the lines will be skew, e.g. not intersecting and not parallel. Thus the point $q$ along the beam trajectory, which has the shortest distance to the centroid's line of sight, is adopted as the point where the laser illuminates the surface.

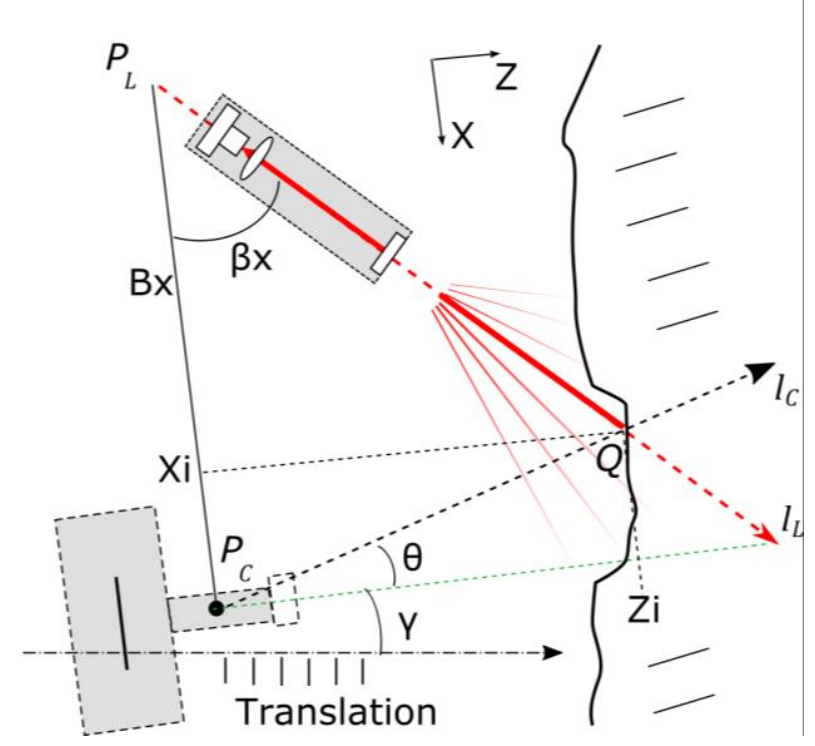

Figure 16: Sketch of the structured light system together with the translation stage and sample target.

Two lines, $l_{L}$ and $l_{C}$, are formulated to describe the trajectory of the laser beam and line of sight extended from the centroid, respectively,

$$
\begin{aligned}
& l_{L}=P_{L}+t_{L} v_{L}, \\
& l_{c}=P_{c}+t_{c} v_{c},
\end{aligned}
$$

where $p_{L}=\left[\begin{array}{lll}B_{x} & B_{y} & 0\end{array}\right]^{T}, p_{C}=\left[\begin{array}{lll}0 & 0 & 0\end{array}\right]^{T}$, and both $t_{L}$ and $t_{c}$ are real valued scalars. The vector $v_{L}$ is given by rotating the boresight vector $\left[\begin{array}{lll}0 & 0 & 1\end{array}\right]^{T}$ sequentially about the $x$ and $y$ axes using a Direction Cosine Matrix defined by the angles $\beta_{x}$, and $\beta_{y}$. The line of sight vector is given as $v_{C}=$ $[x / f, y / f, 1]^{T}$ where $x$ and $y$ are the centroid coordinates that are corrected for principal point offset and lens distortion, and $f$ is the focal length.

The vector $n=v_{L} \times v_{C}$ describes the direction of the shortest distance between the two lines and is perpendicular to both $v_{L}$ and $v_{C}$, and is pointing towards the reader out of the paper, at the point $Q$, as shown in Figure 16. This vector is utilized to formulate a plane extended by $v_{C}$ and $n$ which has the normal $n_{p}=v_{C} \times n$.

The 3D world coordinate, $Q$, where the laser beam illuminates the targeted surface, is then defined as the intersection between $l_{L}$ and the aforementioned plane, e.g. the point along the laser beam trajectory closest to the line of sight extended from the centroid

$$
Q=P_{L}+\frac{n_{p} \cdot\left(P_{C}-P_{L}\right)}{n_{p} \cdot v_{L}} v_{L} .
$$

This formulation eliminates the centroid error perpendicular to the trajectory of the laser, seen from the camera, leaving only the error along the trajectory that will be visible in the estimate of the distance.

\section{CALIBRATION}

The calibration is performed by translating the structured light system, relative to a plane calibration target with uniform albedo. The structure is translated at a fixed translation step, $\delta_{\text {step }}=0.5 \mathrm{~mm}$ using a translation stage with an on-axis accuracy of $5 \mu \mathrm{m}$. At each position a centroid measurement is logged and corrected for radial and tangential lens distortion.

A least square cost function is formulated based on the measured and modelled centroid coordinates, $q_{i}$ and $p_{i}$, respectively,

$$
r=\sum_{i=1}^{n} r_{i}^{2}
$$

where

$$
r_{i}=\sqrt{\left(p_{i_{x}}-q_{i_{x}}\right)^{2}+\left(p_{\mathrm{i}_{\mathrm{y}}}-q_{i_{y}}\right)^{2}} .
$$

The modeled centroids are given by a pinhole projection

$$
p_{i}=\left[f \frac{X_{i}}{Z_{i}}, f \frac{Y_{i}}{Z_{i}}\right] .
$$


The world coordinates $X_{i}, Y_{i}, Z_{i}$ of the modelled centroids are formulated by the baseline $B$ and the angle $\beta$

$$
\begin{aligned}
& X_{i}=B_{x}-\frac{Z_{i}}{\tan \left(\beta_{x}\right)}, \\
& Y_{i}=B_{y}-\frac{Z_{i}}{\tan \left(\beta_{y}\right)}, \\
& Z_{i}=Z_{0}+d Z_{i},
\end{aligned}
$$

where $d Z_{i}=i \cdot \delta_{\text {step }}$. With the camera's optical axis roughly aligned with the translation, the actual increment along boresight is given by $\delta_{z}=\delta_{\text {step }} / \cos (\gamma)$, where $\gamma$ is the angle between the camera boresight and the direction of the translation stage. Given an initial guess on the variables $B_{x}, B_{y}, \beta_{x}, \beta_{y}, Z_{0}$ and $\gamma$ a minimum for the cost function is estimated using a six dimensional Nelder-Mead optimization [38]. Figure 17 shows the increment of the centroid coordinates for the calibration measurements together with the least square fitted model. Figure 18 shows the centroid and distance residual as a function of the position of the instrument structure. The centroid is accurate to 0.2241 pixels $(1 \sigma)$ and the distance is accurate to 15.85 $\mu \mathrm{m}(1 \sigma)$. Note that the residual of the $x$ coordinate of Figure 18 indicates the presence of a sinusoidal bias, which is due to an imperfect calibration surface. For future work more suitable calibration equipment will be applied to improve the overall performance. An image of the calibrated test setup in operation is shown in Figure 19 with the calibrated baseline and angle listed in Table 5 .

The results/calibration presented in this paper is for a structured light system operating at room temperature in a laboratory. However, the structured light system has to operate on the surface of Mars at around $-60^{\circ} \mathrm{C}$. The structured light system presented in this paper is only a prototype. Great attention will be paid to thermal design, minimizing thermal gradients and using low coefficient thermal expansion (CTE) materials for the flight implementation. The plan is to perform only one calibration of the structural light system before launch at room temperature (and allow for the $\sim 0.4 \mathrm{~mm}$ degraded accuracy due to thermal contractions (see details of thermal deformations in Table 2) and allow for up to $0.2 \mathrm{~mm}$ degraded performance due to the laser spot changing positions due to the wavelength shift of the laser). In the further development of the system these error terms will be addressed and tested in a vacuum chamber, verifying the expected behavior. Additionally, the Mars Rover has a calibration target mounted on it. Therefore, it is possible to perform a calibration of the structured light system on the surface of Mars. The calibration target is black covered with 100 white spots to determine the pose of the camera, so the true camera position can be calculated for the calibration of the structured light system.

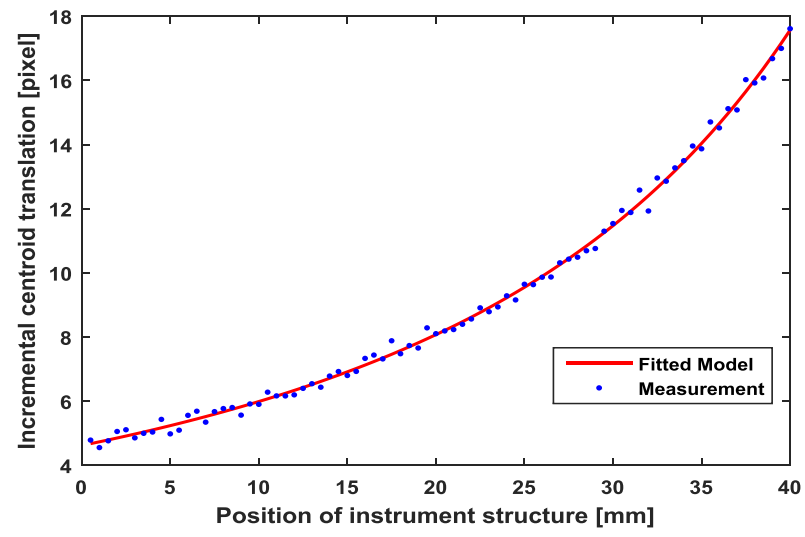

Figure 17: The increment of the consecutive centroid measurements as function of the translation of the structured light system.
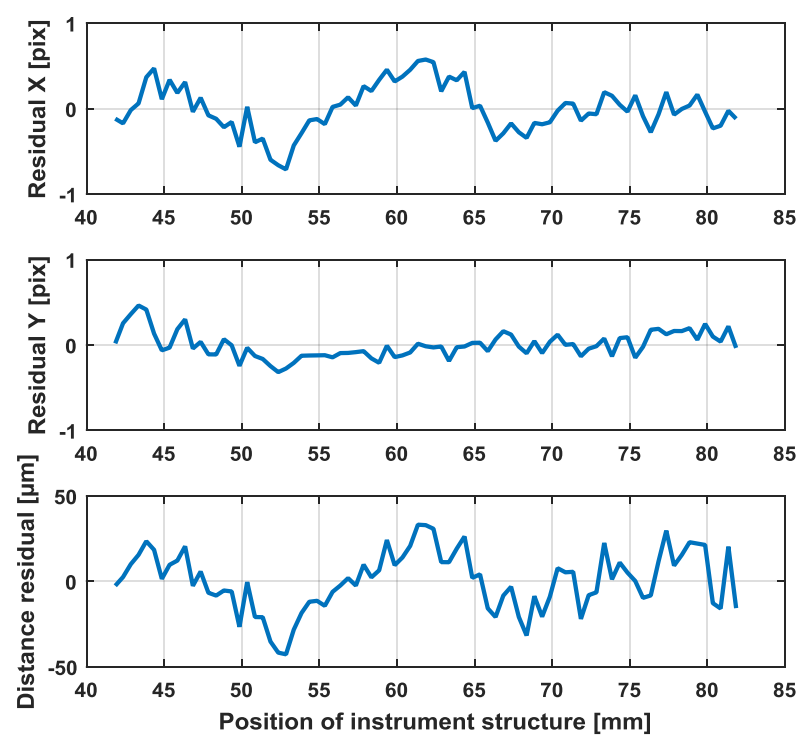

Figure 18: The centroid and corresponding distance residual from the calibration routine determining the calibration parameters of the laser relative to the camera coordinate frame.

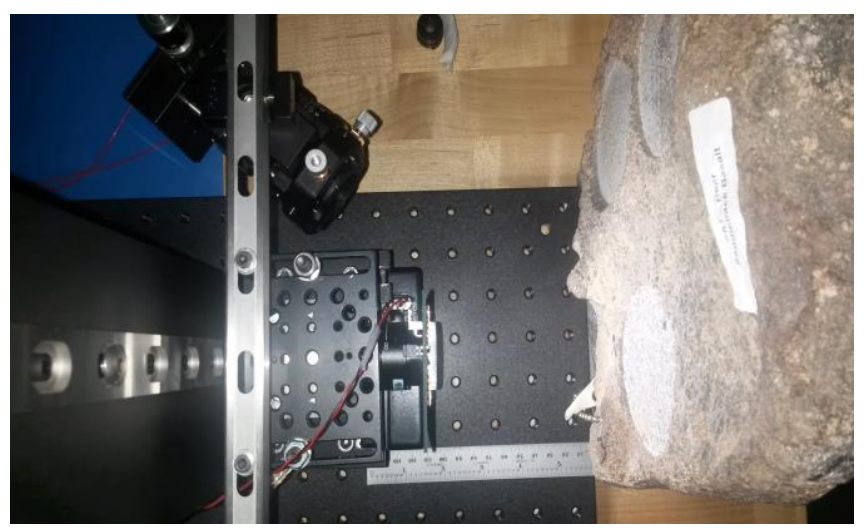

Figure 19: The structured light system consisting of the CCD sensor, laser source (black box at angle at top of image). In this specific image, the system is measuring the distance to an abraded Saddleback Basalt sample.

Table 5: Calibrated system parameters describing the baseline and angle of the structured light system.

\begin{tabular}{ll}
\hline$B_{x}$ & $-43.83 \mathrm{~mm}$ \\
$B_{y}$ & $-1.94 \mathrm{~mm}$ \\
$\beta_{x}$ & $52.35^{\circ}$ \\
$\beta_{y}$ & $87.90^{\circ}$ \\
\hline
\end{tabular}


VII. HEIGHT PROFILE OF ROCK SAMPLE

To illustrate the performance of the demonstration system, measurements were conducted on a sample of an abraded Saddleback Basalt to measure the profile of the surface. Figure 21 shows the measured profile of the sample shown in Figure 20. The linear measurements along the $x$ axis represent the flat abraded area. The jump in the middle represents a small dimple in the sample. The jump of $2 \mathrm{~mm}$ on the left side is where the laser spot moves outside the abraded area.

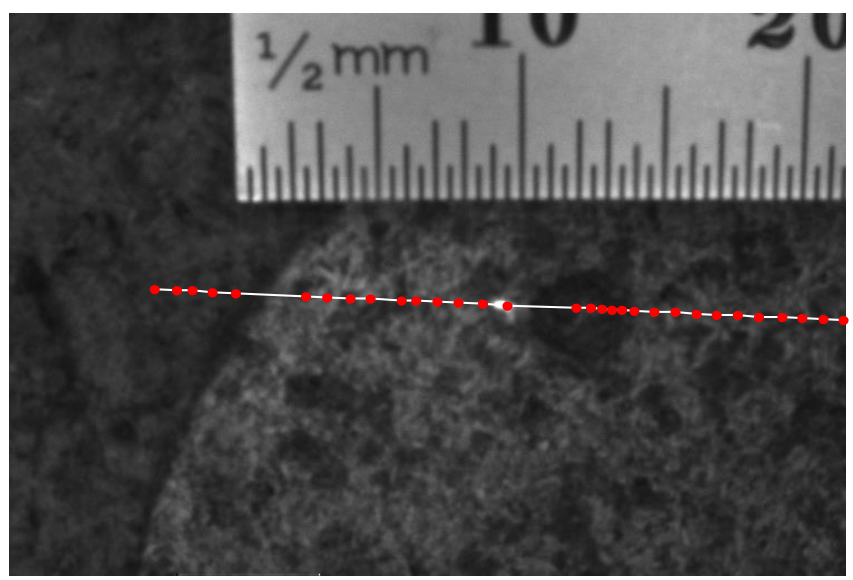

Figure 20: Sample of abraded Saddleback Basalt surface sample used for profile measurements. The edge of abraded area seen on the left side and a dimple in the abraded area is located to the right along the scanning line.

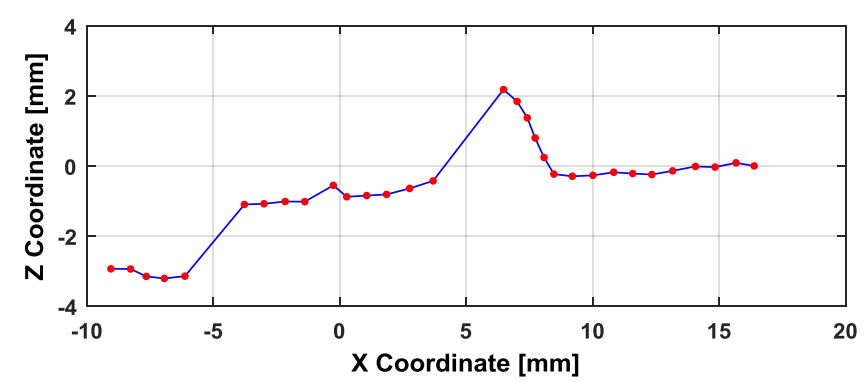

Figure 21: The measured profile of an abraded Saddleback Basalt surface sample. The profile shows a flat surface along the $x$ axis. In the center a dimple in the surface sample is detected, with a depth of $\sim 2.4$ $\mathrm{mm}$. On the far left the laser spot has moved outside the abraded area.

\section{SUMMARY}

A structured light system to guide the positioning of a scientific instrument for robotic planetary exploration is presented. The accuracy of the centroiding of the individual laser spots as well as identification of the laser spots is discussed. The accuracy of the prototype system is $\sim 0.3$ pixels $(1 \sigma)$ over the full operational range of the system. The calibrated distance accuracy is better than 16 microns $(1 \sigma)$ over the full range of operation. It should be emphasized that only a demonstration system has been tested. The real structured light system will be operating more than $80^{\circ} \mathrm{C}$ colder than where it is calibrated. It is expected that thermal induced errors will degrade the overall accuracy of a future system on Mars to $\sim 0.7 \mathrm{~mm}$ accuracy.

\section{ACKNOWLEDGEMENT}

The research described in this paper was carried out at the Technical University of Denmark and the Jet Propulsion Laboratory, California Institute of Technology and was sponsored by the Technical University of Denmark and the National Aeronautics and Space Administration.

\section{REFERENCES}

[1] Jet Propulsion Laboratory, California Institute of Technology, "Mars 2020," [Online]. Available: http://mars.nasa.gov/mars2020/. [Accessed 7 September 2015].

[2] Allwood, A., Clarke, B., Elam, T., Flannery, D., Foote, M., Hurowitz, J., Knowles, E., and Wade, L., "Texturespecific elemental analysis of rocks and soils with PIXL: The Planetary Instrument for X-ray Lithochemistry on Mars 2020," in Proceedings of the IEEE Aerospace Conference, Big Sky MT, 2015.

[3] Allwood, A. C., Wade, L., Hurowitz, J., Hodyss, R., Flannery, D., "Seeking Ancient Microbial Biosignatures with PIXL on Mars 2020," in American Geophysical Fall Meeting, 2014

[4] Goldberg, S. B., Mainmone, M. W., and Matthies, L., "Stereo Vision and Rover Navigation Software for Planetary Exploration," Proceedings, IEEE Aerospace Conference, vol. 5, pp. 2025-2036, 2002.

[5] Ens, J. and Lawrence, P., "An Investigation of Methods for Determining Depth from Focus," IEEE Transactions on Pattern Analysis and Machine Intelligence, vol. 15, no. 2, pp. 97-108, 1993.

[6] Levin, A., Fergus, R., Durand, F. and Freeman, W. T. "Image and Depth from a Conventional Camera with a Coded Aperture," in Proceedings of ACM SIGGRAPH, 2007.

[7] Kleinschmidth, P., Magori, V., "Ultrasonic RoboticSensors for Exact Short Range Distance Measurement and Object Identification," IEEE 1985 Ultrasonic Symposium, pp. 457-462, 1985.

[8] Winder. A. A., "II. Sonar System Technology," IEEE Transactions on Sonics and Ultrasonics, vol. 22, no. 5 , pp. 291-332, 1975.

[9] Pollard, B. D. and Sadowy, G., "Next Generation Millimeter-wave Radar for Safe Planetary Landing," IEEE Aerospace Conference, pp. 1213-1219, 2005. 
[10] Norgia, M., Giuliani, G. and Donati, S., "Absolute Distance Measurement With Improved Accuracy Using Laser Diode Self-Mixing Interferometry in a Close Loop," IEEE Transactions on Instrumentation and Measurement, vol. 56, no. 5, pp. 1894-1900, 2007.

[11] Dubovitsky, S., Lay, O. P., Peters, R. D. and Liebe, C. C., "Absolute Optical Metrology: Nanometers to Kilometers," 2005 Pacific Rim Conference on Lasers and Electro-Optics, pp. 1581-1583, 2005.

[12] Amman, M., Bosch, T., Lescure, M., Myllylä, R. and Rioux, M., "Laser Ranging: A Critical Review of Usual Techniques for Distance Measurement," Optical Engineering, vol. 40, no. 1, pp. 10-19, 2001.

[13] Dorsch, R. G., Häusler, G. and Hermann, J. M., "Laser Triangulation: Fundamental Uncertainty in Distance Measurement," Applied Optics, vol. 33, no. 7, pp. 1306-1314, 1994.

[14] Zhang, Z., "Microsoft Kinect Sensor and Its Effects," IEEE Multimedia, vol. 19, no. 2, pp. 4-10, 2012.

[15] Mutto, C. D., Zanuttigh, P. and Cortelazzo, G. M., "Time-Of-Flight Cameras and Microsoft Kinect(Tm)," Springer Publishing Company, Incorporated, 2012.

[16] Lange, R. and Seitz, P., "Solid-State Time-Of-Flight Range Camera," IEEE Journal of Quantum Electronics, vol. 37, no. 3, pp. 390-397, 2001.

[17] Posdamer, J. L., and Altschuler, M. D., "Surface Measurement by Space-Encoded Projected Beam Systems," Computer Graphics and Image Processing, vol. 18 , no. 1, pp. 1-17, 1982.

[18] Geng, J., "Structured Light 3D Surface Imaging: A Tutorial," Advances in Optics and Photonics, vol. 3, no. 2, pp. 128-160, 2011.

[19] Kawano, I., Mokuno, M., Kasai, T. and Suzuki, T., "Result of Autonomous Rendezvous Docking Experiment of Engineering Test Satellite-VII," Journal of Spacecraft and Rockets, vol. 38, no. 1, pp. 105-111, 2001.

[20] Liebe, C. C., Alexander, J., Aung, M., Goldberg, H., Johnson, A., Lam, R., Maize, E., Meras, P., Montgomery, J., Palacios, P., Spiers, G., Wilson, M., "Field Testing of Lunar Access and Naviation Device (LAND)," IEEE Aerospace Conference, Big Sky, MT., pp. 1-12, 2007.

[21] Mishkin, A. H., Morrison, J. C., Nguyen, T. T., Stone, H. W., Cooper, B. K. and Wilcox, B. H., "Experiences with Operations and Autonomy of the Mars Pathfinder Microrover," IEEE Aerospace Conference Proceedings, vol. 2, pp. 337-351, 1998.

[22] Matthies, L., Balch, T., Wilcox, B., "Fast Optical Hazard Detection for Planetary Rovers using Multiple Spot Laser Triangulation," in IEEE Int. Conference on Robotics and Automation, Albuquerque, 1997.

[23] Baumgartner, E. T., Bonitz, R. G., Melko, J. P., Shiraishi, L. R., Chris Leger, P., "The Mars Exploration Rover Instrument Positioning System," in IEEE Aerospace Conference, Big Sky, 2005.

[24] Jørgensen, J. L., Denver, T., Mauizio, B., Jørgensen, P. S., "The Micro ASC, a Miniature Star Tracker," Small Satellites for Earth Observation, 4th International Symposium of the International Academy of Astronautics, pp. 157-162, 2003.

[25] Rybicki, G. B., Lightman, A. P., Radiative Processes in Astrophysics, Wiley-VCH, 1994.

[26] Haberle, R. M., McKay, C. P., Pollack, J. B., Gwynne, O. E., Atkinson, D. H., Appelbaum, J., Landis, G. A., Zurek, R. W., Flood, D. J., "Atmospheric Effects On The Utility Of Solar Power On Mars," in Resources of Near-Earth Space, vol. 117 (256 pp), Tuscon, University of Arizona Press, 1993, pp. 845-885.

[27] Vincendon, M., "Mars Surface Phase Function Constrained by Orbital Observations," Planetary and Space Science, vol. 76, pp. 87-95, 2013.

[28] Vaucouleurs, G. D., Physics of Planet Mars, London: Faber and Faber Limited, 1952.

[29] Edwards, P. H., Bridges, J. C., Wiens, R., Anderson, R., Dyar, D., Fisk, M., et al., "Basalt-trachybasalt samples in GaleCrater, Mars," Meteoritics \& Planetary Science, vol. 52, no. 11, 2017.

[30] Yen, A. S., Ming, D. W., Vaniman, D. T., Gellert, R., Blake, D. F., Morris, R. V., et al., "Multiple stages of aqueous alteratinos along fracturs in mudstone and sandstone strata in Gale Crater, Mars," Earth and Planetary Science Letters, vol. 471, pp. 186-198, 2017.

[31] Rampe, E. B., Ming, D. W., Blake, D. F, Bristow, T. F., Chipera, S. J., Grotzinger, J. P., et al., "Mineralogy of an ancient lacustrine mudstone succession from the Murray formation, Gale crater, Mars," Earth and Planetary Science Letters, vol. 471, pp. 172-185, 2017.

[32] Banham,S. G., Gupta, S., Rubin, D. M., Watkins, J. A., Sumner, D. Y., Grotzinger, J. P., et al., "Anatomy of an 
Ancient Aeolian Sandstone on Mars; The Stimson Formation in Gale Crater," in AAS/Division for Planetary Sciences Meeting, 2017.

[33] Bass, M., Stryland, E. W. V., Williams, D. R., Wolfe, W. L., Handbook of Optics Vol. 2 Devices, Measurements, and Properties, McGraw-Hill, 1995.

[34] N. G. Barlow, Mars: An Introduction to its Interior, Surface and Atmosphere, New York: Cambridge University Press, 2008.

[35] Bennet, W. R., "Spectra of Quantized Signals," Bell System Technical Journal Vol. 27, pp. 446-471, 1948.

[36] Hartley, R., Zisserman, A., Multiple View Geometry in Computer Vision, Second Edition, Cambridge University Press, 2004.

[37] Horn, B. K. P., Robot Vision, Cambridge: The MIT Press, 1986.

[38] Nocedal, J., Wright, S., Numerical Optimization, Second Edition, New York: Springer Science+Bussiness Media, LLC, 2006.

[39] Liebe, C. C., Coste, K., "Distance Measurement Utilizing Image-Based Triangulation," Sensors Journal, IEEE, vol. 13, no. 1, pp. 234-244, January 2013.

[40] Acord. K., Parker, M., Dodd, E., "High frequency vibration and high gravity force shock testing for potential Mars Sample Return," in Aerospace Conference IEEE, 2015.

[41] Salvi, J., Pagès, J., Batlle, J., "Pattern codification strategies in structured light systems," Pattern Recognition, no. 37, pp. 827-849, 2004.

[42] Liebe, C. C., Padgett, C., Chapsky, J., Wilson, D., Brown, K., Jerebets, S., Goldberg, H., Schroeder, J., "Spacecraft Hazard Avoidance Utilizing Structured Light," in Aerospace Conference, IEEE, Big Sky, MT, 2006.

[43] Sansoni, G., Trebeschi, M., Docchio, F., "State-of-TheArt And Applications of 3D Imaging Sensors in Industry, Cultural Heritage, Medicine, and Criminal Investigations," Sensors, pp. 568-601, 2009.

[44] Liebe, C. C., Padgett, C., Chang, J., "Three Dimensional Imaging Utilizing Structured Light," in Aerospace Conference Proceedings, IEEE, Big Sky, MT, 2004.
[45] Blais, F., "A review of 20 years of range sensors development," J. Electronic Imaging 13, pp. 231-240, 2004.

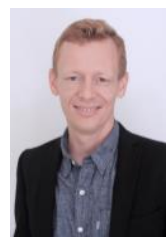

BIOGRAPHIES

David Arge Klevang Pedersen received the M.S.E.E. degree in 2011 and the Ph.D. degree in 2016 from the Department of Measurement and Instrumentation Systems at DTU Space, Technical University of Denmark. Currently, he is an assistant professor at DTU Space. His research is focused on visual tracking of non-cooperative space borne targets.

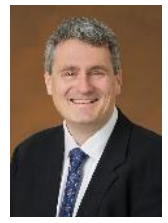

Carl Christian Liebe received the M.S.E.E. degree in 1991 and the Ph.D. degree in 1994 from the Department of Electro-physics, Technical University of Denmark. Since 1997, he has been an employee at the Jet Propulsion Laboratory, California Institute of Technology. He is a Principal engineer and supervisor of the GN\&C Hardware and Testbed Development Group. He has led the development of several metrology systems for space flight.

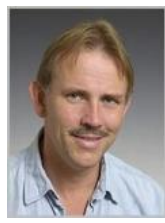

John Leif Jørgensen is professor and head of Measurement and Instrumentation department at the DTU Space, the National Space Institute, at Technical University of Denmark. Professor Jørgensen is an expert in advanced measurement systems and his research focus is on development of optical guidance and navigation systems. 